Acta Crystallographica Section E

Structure Reports

Online

ISSN 1600-5368

\section{1,4-Bis(4-bromo-2,6-diisopropylphenyl)- 1,4-diazabuta-1,3-diene}

\author{
Ilia A. Guzei,* Nicholas J. Hill and Matthew R. Van Hout \\ Department of Chemistry, University of Wisconsin-Madison, 1101 University Ave, \\ Madison, WI 53706, USA \\ Correspondence e-mail: iguzei@chem.wisc.edu
}

Received 11 November 2009; accepted 25 November 2009

Key indicators: single-crystal X-ray study; $T=300 \mathrm{~K}$; mean $\sigma(\mathrm{C}-\mathrm{C})=0.010 \AA$; $R$ factor $=0.069 ; w R$ factor $=0.199 ;$ data-to-parameter ratio $=16.1$.

The molecule of the title compound, $\mathrm{C}_{26} \mathrm{H}_{34} \mathrm{Br}_{2} \mathrm{~N}_{2}$, lies on a crystallographic inversion center and hence the two imine groups are $s$-trans. The dihedral angle between the central 1,4diazabuta-1,3-diene unit and the attached substituted phenyl ring is $88.4(7)^{\circ}$. The structure features a $\mathrm{C}-\mathrm{H} \cdots \mathrm{N}$ close contact. The crystal selected for this study proved to be a nonmerohedral twin with a minor component of 21.8 (3)\%.

\section{Related literature}

1,4-diaza-1,3-butadiene (DAB) ligands containing sterically demanding $N$-substituents have proved to be versatile platforms for stabilizing $s$ - and $p$-block atoms in unusual oxidation states or coordination geometries, see: Baker et al. (2008); Hill et al. (2009); Liu et al. (2009); Martin et al. (2009); Segawa et al. (2008). The title compound was prepared as part of our continuing studies on the chemistry of $N$-heterocyclic silylenes and germylenes, see: Hill et al. (2005); Naka et al. (2004); Tomasik et al. (2009). For the use of DAB ligands in olefin polymerization catalysis, see: Ittel et al. (2000); Jung et al. (2007). For related structures, see: (2003); Müller et al. (2003); Schaub et al. (2006); Berger et al. (2001); Laine et al. (1999). For the preparation of 4-bromo-2,6-di-iso-propyl aniline, see: Liu et al. (2005). For a description of the Cambridge Structural Database, see: Allen (2002).

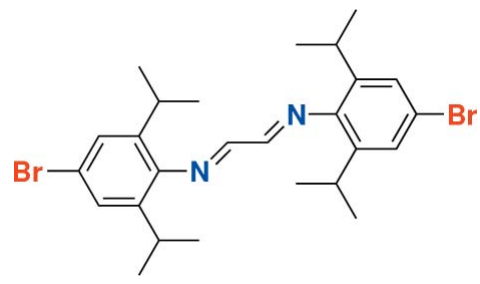

\section{Experimental}

$$
\begin{aligned}
& \text { Crystal data } \\
& \mathrm{C}_{26} \mathrm{H}_{34} \mathrm{Br}_{2} \mathrm{~N}_{2} \\
& M_{r}=534.37 \\
& \text { Monoclinic, } P 2_{1} / c \\
& a=8.961(3) \AA \\
& b=17.848(7) \AA \\
& c=8.620(3) \AA \\
& \beta=104.260(11)^{\circ}
\end{aligned}
$$

\section{Data collection}

Bruker SMART X2S diffractometer Absorption correction: multi-scan (TWINABS; Bruker, 2007) $T_{\min }=0.103, T_{\max }=0.428$

\section{Refinement}

$R\left[F^{2}>2 \sigma\left(F^{2}\right)\right]=0.069$

$w R\left(F^{2}\right)=0.199$

$S=1.04$

2286 reflections

$$
\begin{aligned}
& V=1336.2(8) \AA^{3} \\
& Z=2 \\
& \text { Mo K } \alpha \text { radiation } \\
& \mu=3.05 \mathrm{~mm}^{-1} \\
& T=300 \mathrm{~K} \\
& 0.43 \times 0.35 \times 0.29 \mathrm{~mm}
\end{aligned}
$$

\section{Table 1}

Hydrogen-bond geometry $\left(\AA{ }^{\circ}\right)$.

\begin{tabular}{lllll}
\hline$D-\mathrm{H} \cdots A$ & $D-\mathrm{H}$ & $\mathrm{H} \cdots A$ & $D \cdots A$ & $D-\mathrm{H} \cdots A$ \\
\hline $\mathrm{C} 8-\mathrm{H} 8 \cdots \mathrm{N} 1$ & 0.98 & 2.40 & $2.880(9)$ & 109 \\
\hline
\end{tabular}

2286 measured reflections 2286 independent reflections 1585 reflections with $I>2 \sigma(I)$ $R_{\text {int }}=0.110$

\section{2 parameters}

$\mathrm{H}$-atom parameters constrained

$\Delta \rho_{\max }=0.53{\mathrm{e} \AA^{-3}}^{-3}$

$\Delta \rho_{\min }=-0.60{\mathrm{e} \AA^{-3}}^{-3}$

Data collection: GIS (Bruker, 2009); cell refinement: SAINT (Bruker, 2007); data reduction: $S A I N T$; $\operatorname{program}(\mathrm{s})$ used to solve structure: SHELXTL (Sheldrick, 2008); program(s) used to refine structure: SHELXTL and OLEX2 (Dolomanov et al., 2009); molecular graphics: SHELXTL and $O L E X 2$; software used to prepare material for publication: SHELXTL, OLEX2 (Dolomanov et al., 2009), publCIF (Westrip, 2009) and modiCIFer (Guzei, 2007).

We gratefully acknowledge Bruker AXS sponsorship of this publication.

Supplementary data and figures for this paper are available from the IUCr electronic archives (Reference: BX2248).

\title{
References
}

Allen, F. H. (2002). Acta Cryst. B58, 380-388.

Baker, R. J., Jones, C., Mills, D. P., Pierce, G. A. \& Waugh, M. (2008). Inorg. Chim. Acta, 361, 427-435.

Berger, S., Baumann, F., Scheiring, T. \& Kaim, W. (2001). Z. Anorg. Allg. Chem. 627, 620-630.

Bruker (2007). TWINABS and SAINT. Bruker AXS Inc., Madison, Wisconsin, USA.

Bruker (2009). GIS. Bruker AXS Inc., Madison, Wisconsin, USA.

Dolomanov, O. V., Bourhis, L. J., Gildea, R. J., Howard, J. A. K. \& Puschmann, H. (2009). J. Appl. Cryst. 42, 339-341.

Guzei, I. A. (2007). modiCIFer. Molecular Structure Laboratory, University of Wisconsin-Madison, Madison, Wisconsin, USA.

Hill, N. J., Moser, D. F., Guzei, I. A. \& West, R. (2005). Organometallics, 24, 3346-3349.

Hill, N. J., Vargas-Baca, I. \& Cowley, A. H. (2009). Dalton Trans. pp. 240-253. Ittel, S. D., Johnson, L. K. \& Brookhart, M. (2000). Chem. Rev. 100, 1169-1203. Jung, I. G., Seo, J., Chung, Y. K., Shin, D. M., Chun, S.-H. \& Son, S. U. (2007). J. Polym. Sci. Part A Polym. Chem. 45, 3042-3052.

Laine, T. V., Klinga, M., Maaninen, A., Aitola, E. \& Leskela, M. (1999). Acta Chem. Scand.. 53, 968-973. 
Liu, H.-R., Gomes, P. T., Costa, S. I., Duarte, M. T., Branquinho, R., Fernades, A. C., Chein, J. W., Singh, R. P. \& Marques, M. M. (2005). J. Organomet. Chem. 690, 1314-1322.

Liu, Y., Li, S., Yang, X.-J., Yang, P. \& Wu, B. (2009). J. Am. Chem. Soc. 131, 4210-4211.

Martin, C. D., Jennings, M. C., Ferguson, M. J. \& Ragogna, P. J. (2009). Angew. Chem. Int. Ed. 48, 2210-2213.

Müller, T., Schrecke, B. \& Bolte, M. (2003). Acta Cryst. E59, o1820-o1821.
Naka, A., Hill, N. J. \& West, R. (2004). Organometallics, 23, 6330-6332.

Schaub, T. \& Radius, U. (2006). Z. Anorg. Allg. Chem. 632, 807-813.

Segawa, Y., Suzuki, Y., Yamashita, M. \& Nozaki, K. (2008). J. Am. Chem. Soc. 130, 16069-16079.

Sheldrick, G. M. (2008). Acta Cryst. A64, 112-122.

Tomasik, A. C., Hill, N. J. \& West, R. (2009). J. Organomet. Chem. 694, 2122 2125.

Westrip, S. P. (2009). publCIF. In preparation. 


\section{supporting information}

Acta Cryst. (2010). E66, o40-o41 [doi:10.1107/S1600536809050843]

\section{1,4-Bis(4-bromo-2,6-diisopropylphenyl)-1,4-diazabuta-1,3-diene}

\section{Ilia A. Guzei, Nicholas J. Hill and Matthew R. Van Hout}

\section{S1. Comment}

1,4-diaza-1,3-butadiene (DAB) ligands bearing bulky aryl or alkyl groups on the nitrogen atoms have proven to be versatile platforms for stabilizing s- and $p$-block atoms in unusual oxidation states or coordination geometries (Baker $e t$ al. (2008); Hill et al. (2009); Liu et al. (2009); Martin et al. (2009); Segawa et al. (2008)). The title compound, (I), was prepared as part of our continuing studies upon the chemistry of N-heterocyclic silylenes and germylenes (Hill et al. (2005); Naka et al. (2004); Tomasik et al. (2009)), the silicon(II) and germanium(II) analogues of the well known Arduengo N-heterocyclic carbenes. DAB ligands are ideal in this regard since their stereo-electronic properties are easily tuned by alteration of the $\mathrm{N}-$ and $\mathrm{C}$-substituents.

DAB ligands have also been used extensively in d-block coordination chemistry, particularly within the field of olefin polymerization catalysis (Ittel et al. 2000). Jung et al. (2007) recently used the title compound as a precursor to an Nheterocyclic carbene in the synthesis of a catalytically active cationic $\left(\eta^{3}\right.$-allyl)(NHC)palladium complex.

The molecule of (I) resides on a crystallographic inversion center and hence the two imine groups are $s$-trans. The dihedral angle between the central 1,4-diazabuta-1,3-diene moiety and the attached substituted phenyl ring is $88.4(7)^{\circ}$. The molecular symmetry approaches $\mathrm{C}_{2 \mathrm{~h}}$, however, the positions of the isopropyl groups break the mirror plane symmetry: both $\mathrm{H}$ atoms on the tertiary $\mathrm{C}$ atoms of the two symmetry-independent ${ }^{\text {Pr }} \mathrm{Pr}$ groups point toward atom N1, but reside on the opposite sides of the phenyl ring. This is illustrated with two disparate but "would be equivalent" torsion angles, one for each ${ }^{\mathrm{P}} \mathrm{Pr}$ group: $\mathrm{C} 2-\mathrm{C} 3-\mathrm{C} 8-\mathrm{C} 9\left(-96.5(8)^{\circ}\right)$ and $\mathrm{C} 2-\mathrm{C} 7-\mathrm{C} 11-\mathrm{C} 13\left(163.6(7)^{\circ}\right)$. This geometry differs from that of the unbrominated congener of (I), 1,4-bis(2,6-diisopropyl-phenyl)-1,4-diazabuta-1,3-diene, (II). For related structures, see: Müller et al. (2003), Schaub et al. (2006). Compound (II), structurally characterized at $173 \mathrm{~K}$ by Berger et al. (2001) and at $193 \mathrm{~K}$ by Laine et al.(1999), crystallizes with the molecule of (II) on an inversion center. The $\mathrm{H}$ atoms of the tertiary $\mathrm{C}$ atom of the isopropyl groups point toward the $\mathrm{N}$ atom and, in contrast to (I), are located on the same side of the phenyl ring. The overall symmetry of (II) is much closer to $\mathrm{C}_{2 \mathrm{~h}}$ as the ${ }^{i} \operatorname{Pr}$ groups are oriented very similarly: in the $193 \mathrm{~K}$ structure of (II) two "would be equivalent" $\mathrm{Me}-\mathrm{C}(\mathrm{H})-\mathrm{C}-\mathrm{C}(\mathrm{N})$ torsion angles measured 144.6 and $145.4^{\circ}$. The $\mathrm{C}-\mathrm{Br}$ distance of 1.897 (6) $\AA$ is in excellent agreement with the value of 1.899 (11) $\AA$ obtained by averaging $2303 \mathrm{C}-\mathrm{Br}$ bond lengths from 1736 relevant compounds reported to the Cambridge Structural Database (Allen, 2002).

\section{S2. Experimental}

4-Bromo-2,6-di-iso-propyl aniline was prepared according to the literature procedure (Liu et al. 2005). To a stirred solution of 4-bromo-2,6-di-iso-propyl aniline $(3.0 \mathrm{~g}, 11.71 \mathrm{mmol})$ in methanol $\left(40 \mathrm{~cm}^{3}\right)$ containing 4 drops of formic acid was added glyoxal ( $0.85 \mathrm{~g}, 5.80 \mathrm{mmol}, 40 \%$ aqueous soln.) slowly dropwise. The reaction mixture was stirred for $24 \mathrm{~h}$ at room temperature, filtered, and the precipitate washed with cold $\mathrm{MeOH}(2 \times 10 \mathrm{~mL})$. This yellow solid was dried in vacuo and recrystallized from EtOH to give a crop of pale yellow needles suitable for X-ray diffraction analysis. Yield 
$3.53 \mathrm{~g}, 56 \%$.

${ }^{1} \mathrm{H}-\mathrm{NMR}\left(\mathrm{CD}_{2} \mathrm{Cl}_{2}, 300 \mathrm{MHz}\right): \delta 1.19\left(\mathrm{~d},{ }^{3} J=6.9 \mathrm{~Hz}, 24 \mathrm{H}, \mathrm{CH}_{3}\right), 2.91$ (sept, $\left.{ }^{3} J=6.8 \mathrm{~Hz}, 4 \mathrm{H}, \mathrm{CH}\right), 7.31(\mathrm{~s}, 4 \mathrm{H}$, aromatic), 8.07 (s, 2H, CH); ${ }^{13} \mathrm{C}\left\{{ }^{1} \mathrm{H}\right\}-\mathrm{NMR}\left(\mathrm{CD}_{2} \mathrm{Cl}_{2}, 75 \mathrm{MHz}\right): \delta 22.80,28.43,119.42,126.79,139.29,147.52,163.97$.

\section{S3. Refinement}

All H-atoms were placed in idealized locations with $\mathrm{C}-\mathrm{H}$ distances $0.93-0.98 \AA$ and refined as riding with appropriate thermal displacement coefficients $U_{\text {iso }}(\mathrm{H})=1.2$ or 1.5 times $U_{\text {eq }}$ (bearing atom). The crystal of (I) selected for this study proved to be a non-merohedral twin. The two twin components are related by a $179.9^{\circ}$ rotation about the [001] direction in reciprocal space with the minor component contribution of $21.8(3) \%$.

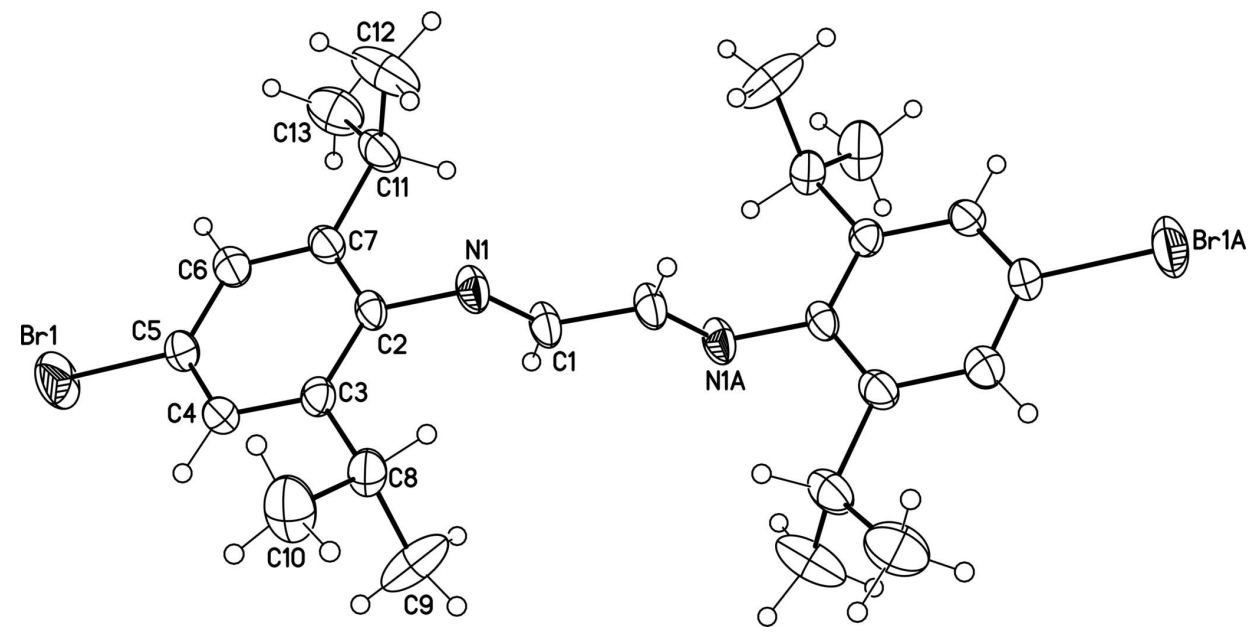

\section{Figure 1}

Molecular structure of (I). The thermal ellipsoids are shown at 30\% probability level. Atoms labeled with the suffixes A and unlabeled are generated by the symmetry operation $(-x+1,-y+1,-z+1)$.

\section{1,4-Bis(4-bromo-2,6-diisopropylphenyl)-1,4-diazabuta-1,3-diene}

Crystal data

$\mathrm{C}_{26} \mathrm{H}_{34} \mathrm{Br}_{2} \mathrm{~N}_{2}$

$M_{r}=534.37$

Monoclinic, $P 2_{1} / c$

Hall symbol: $-\mathrm{P} 2 \mathrm{ybc}$

$a=8.961(3) \AA$

$b=17.848(7) \AA$

$c=8.620(3) \AA$

$\beta=104.260(11)^{\circ}$

$V=1336.2(8) \AA^{3}$

$Z=2$

\section{Data collection}

Bruker SMART X2S

diffractometer

Radiation source: micro-focus sealed tube

Doubly curved silicon crystal monochromator

$\omega$ scans

Absorption correction: multi-scan

(TWINABS; Bruker, 2007)

$T_{\min }=0.103, T_{\max }=0.428$
$F(000)=548$

$D_{\mathrm{x}}=1.328 \mathrm{Mg} \mathrm{m}^{-3}$

Mo $K \alpha$ radiation, $\lambda=0.71073 \AA$

Cell parameters from 999 reflections

$\theta=2.3-24.8^{\circ}$

$\mu=3.05 \mathrm{~mm}^{-1}$

$T=300 \mathrm{~K}$

Block, yellow

$0.43 \times 0.35 \times 0.29 \mathrm{~mm}$

2286 measured reflections

2286 independent reflections

1585 reflections with $I>2 \sigma(I)$

$R_{\text {int }}=0.110$

$\theta_{\text {max }}=24.8^{\circ}, \theta_{\min }=2.3^{\circ}$

$h=0 \rightarrow 10$

$k=-21 \rightarrow 0$

$l=-10 \rightarrow 9$ 


\section{Refinement}

Refinement on $F^{2}$

Least-squares matrix: full

$R\left[F^{2}>2 \sigma\left(F^{2}\right)\right]=0.069$

$w R\left(F^{2}\right)=0.199$

$S=1.04$

2286 reflections

142 parameters

0 restraints

Primary atom site location: structure-invariant direct methods

Secondary atom site location: difference Fourier map

\section{Special details}

Geometry. All e.s.d.'s (except the e.s.d. in the dihedral angle between two 1.s. planes) are estimated using the full covariance matrix. The cell e.s.d.'s are taken into account individually in the estimation of e.s.d.'s in distances, angles and torsion angles; correlations between e.s.d.'s in cell parameters are only used when they are defined by crystal symmetry. An approximate (isotropic) treatment of cell e.s.d.'s is used for estimating e.s.d.'s involving 1.s. planes.

Refinement. Refinement of $F^{2}$ against ALL reflections. The weighted $R$-factor $w R$ and goodness of fit $S$ are based on $F^{2}$, conventional $R$-factors $R$ are based on $F$, with $F$ set to zero for negative $F^{2}$. The threshold expression of $F^{2}>\sigma\left(F^{2}\right)$ is used only for calculating $R$-factors(gt) etc. and is not relevant to the choice of reflections for refinement. $R$-factors based on $F^{2}$ are statistically about twice as large as those based on $F$, and $R$ - factors based on ALL data will be even larger.

Fractional atomic coordinates and isotropic or equivalent isotropic displacement parameters $\left(\AA^{2}\right)$

\begin{tabular}{lllll}
\hline & $x$ & $y$ & $z$ & $U_{\text {iso }} / U_{\text {eq }}$ \\
\hline Br1 & $1.14981(9)$ & $0.69711(6)$ & $0.27272(12)$ & $0.0957(6)$ \\
N1 & $0.6097(6)$ & $0.5812(3)$ & $0.5079(7)$ & $0.0523(14)$ \\
C1 & $0.5657(8)$ & $0.5148(3)$ & $0.4744(8)$ & $0.0513(17)$ \\
H1 & 0.6173 & 0.4847 & 0.4166 & $0.062^{*}$ \\
C2 & $0.7381(6)$ & $0.6090(3)$ & $0.4544(7)$ & $0.0395(14)$ \\
C3 & $0.7094(7)$ & $0.6469(3)$ & $0.3074(7)$ & $0.0409(14)$ \\
C4 & $0.8336(7)$ & $0.6738(3)$ & $0.2564(7)$ & $0.0456(15)$ \\
H4 & 0.8179 & 0.6992 & 0.1595 & $0.055^{*}$ \\
C5 & $0.9804(7)$ & $0.6630(4)$ & $0.3489(8)$ & $0.0500(16)$ \\
C6 & $1.0083(7)$ & $0.6289(4)$ & $0.4968(8)$ & $0.0529(16)$ \\
H6 & 1.1086 & 0.6245 & 0.5591 & $0.063^{*}$ \\
C7 & $0.8860(8)$ & $0.6012(3)$ & $0.5528(8)$ & $0.0485(16)$ \\
C8 & $0.5477(7)$ & $0.6575(4)$ & $0.2044(9)$ & $0.0536(16)$ \\
H8 & 0.4780 & 0.6500 & 0.2746 & $0.064^{*}$ \\
C9 & $0.5079(12)$ & $0.5984(6)$ & $0.0770(14)$ & $0.119(4)$ \\
H9C & 0.5672 & 0.6062 & -0.0004 & $0.179^{*}$ \\
H9B & 0.4002 & 0.6013 & 0.0251 & $0.179^{*}$ \\
H9A & 0.5309 & 0.5498 & 0.1247 & $0.179^{*}$ \\
C10 & $0.5185(9)$ & $0.7356(5)$ & $0.1375(14)$ & $0.088(3)$ \\
H10C & 0.5496 & 0.7714 & 0.2224 & $0.132^{*}$ \\
H10A & 0.4108 & 0.7416 & 0.0883 & $0.132^{*}$ \\
H10B & 0.5766 & 0.7436 & 0.0590 & $0.132^{*}$ \\
C11 & $0.9157(9)$ & $0.5652(4)$ & $0.7203(8)$ & $0.0626(18)$ \\
& & & &
\end{tabular}

Hydrogen site location: inferred from

$w=1 /\left[\sigma^{2}\left(F_{\mathrm{o}}^{2}\right)+(0.0949 P)^{2}+1.843 P\right]$

where $P=\left(F_{\mathrm{o}}^{2}+2 F_{\mathrm{c}}^{2}\right) / 3$

$(\Delta / \sigma)_{\max }=0.001$

$\Delta \rho_{\min }=-0.60 \mathrm{e} \AA^{-3}$

Extinction correction: SHELXTL (Sheldrick, 2008), $\mathrm{Fc}^{*}=\mathrm{kFc}\left[1+0.001 \mathrm{xFc}^{2} \lambda^{3} / \sin (2 \theta)\right]^{-1 / 4}$

Extinction coefficient: 0.038 (5) 


$\begin{array}{lllll}\text { H11 } & 0.8533 & 0.5195 & 0.7090 & 0.075^{*} \\ \text { C12 } & 0.8601(13) & 0.6152(5) & 0.8316(10) & 0.101(3) \\ \text { H12B } & 0.8573 & 0.5880 & 0.9269 & 0.151^{*} \\ \text { H12A } & 0.7586 & 0.6327 & 0.7808 & 0.151^{*} \\ \text { H12C } & 0.9285 & 0.6572 & 0.8591 & 0.151^{*} \\ \text { C13 } & 1.0834(11) & 0.5415(5) & 0.7895(10) & 0.087(3) \\ \text { H13A } & 1.1158 & 0.5093 & 0.7148 & 0.131^{*} \\ \text { H13B } & 1.0915 & 0.5152 & 0.8884 & 0.131^{*} \\ \text { H13C } & 1.1478 & 0.5852 & 0.8086 & 0.131^{*}\end{array}$

Atomic displacement parameters $\left(\AA^{2}\right)$

\begin{tabular}{lllllll}
\hline & $U^{11}$ & $U^{22}$ & $U^{33}$ & $U^{12}$ & $U^{13}$ & $U^{23}$ \\
\hline Br1 & $0.0555(5)$ & $0.1428(10)$ & $0.0961(8)$ & $-0.0228(5)$ & $0.0324(5)$ & $0.0295(6)$ \\
N1 & $0.067(3)$ & $0.034(3)$ & $0.065(4)$ & $-0.011(2)$ & $0.036(3)$ & $-0.001(2)$ \\
C1 & $0.068(4)$ & $0.035(3)$ & $0.063(4)$ & $-0.014(3)$ & $0.040(4)$ & $-0.007(3)$ \\
C2 & $0.053(3)$ & $0.027(3)$ & $0.046(4)$ & $-0.006(2)$ & $0.026(3)$ & $-0.004(3)$ \\
C3 & $0.046(3)$ & $0.033(3)$ & $0.046(4)$ & $-0.005(3)$ & $0.015(3)$ & $-0.003(3)$ \\
C4 & $0.053(4)$ & $0.046(3)$ & $0.039(3)$ & $-0.006(3)$ & $0.014(3)$ & $0.002(3)$ \\
C5 & $0.046(4)$ & $0.059(4)$ & $0.050(4)$ & $-0.011(3)$ & $0.021(3)$ & $0.000(3)$ \\
C6 & $0.052(3)$ & $0.057(4)$ & $0.050(4)$ & $-0.006(3)$ & $0.014(3)$ & $0.006(3)$ \\
C7 & $0.064(4)$ & $0.042(3)$ & $0.043(4)$ & $-0.006(3)$ & $0.020(3)$ & $0.002(3)$ \\
C8 & $0.046(3)$ & $0.052(4)$ & $0.063(4)$ & $-0.005(3)$ & $0.014(3)$ & $0.007(3)$ \\
C9 & $0.093(7)$ & $0.119(8)$ & $0.117(9)$ & $-0.024(6)$ & $-0.030(6)$ & $-0.045(7)$ \\
C10 & $0.063(5)$ & $0.080(5)$ & $0.115(8)$ & $0.008(4)$ & $0.013(6)$ & $0.039(6)$ \\
C11 & $0.083(5)$ & $0.059(4)$ & $0.052(4)$ & $-0.003(4)$ & $0.027(4)$ & $0.014(4)$ \\
C12 & $0.161(10)$ & $0.094(6)$ & $0.063(6)$ & $0.043(6)$ & $0.058(6)$ & $0.026(5)$ \\
C13 & $0.109(7)$ & $0.081(6)$ & $0.072(6)$ & $0.025(5)$ & $0.025(5)$ & $0.019(5)$ \\
& & & & & & \\
\hline
\end{tabular}

Geometric parameters $\left(\AA,{ }^{\circ}\right)$

\begin{tabular}{llll}
\hline $\mathrm{Br} 1-\mathrm{C} 5$ & $1.897(6)$ & $\mathrm{C} 8-\mathrm{H} 8$ & 0.9800 \\
$\mathrm{~N} 1-\mathrm{C} 1$ & $1.260(7)$ & $\mathrm{C} 9-\mathrm{H} 9 \mathrm{C}$ & 0.9600 \\
$\mathrm{~N} 1-\mathrm{C} 2$ & $1.429(7)$ & $\mathrm{C} 9-\mathrm{H} 9 \mathrm{~B}$ & 0.9600 \\
$\mathrm{C} 1-\mathrm{C} 1^{\mathrm{i}}$ & $1.455(11)$ & $\mathrm{C} 9-\mathrm{H} 9 \mathrm{~A}$ & 0.9600 \\
$\mathrm{C} 1-\mathrm{H} 1$ & 0.9300 & $\mathrm{C} 10-\mathrm{H} 10 \mathrm{C}$ & 0.9600 \\
$\mathrm{C} 2-\mathrm{C} 7$ & $1.393(9)$ & $\mathrm{C} 10-\mathrm{H} 10 \mathrm{~A}$ & 0.9600 \\
$\mathrm{C} 2-\mathrm{C} 3$ & $1.403(8)$ & $\mathrm{C} 10-\mathrm{H} 10 \mathrm{~B}$ & 0.9600 \\
$\mathrm{C} 3-\mathrm{C} 4$ & $1.380(8)$ & $\mathrm{C} 11-\mathrm{C} 12$ & $1.483(11)$ \\
$\mathrm{C} 3-\mathrm{C} 8$ & $1.513(9)$ & $\mathrm{C} 11-\mathrm{C} 13$ & $1.533(12)$ \\
$\mathrm{C} 4-\mathrm{C} 5$ & $1.374(9)$ & $\mathrm{C} 11-\mathrm{H} 11$ & 0.9800 \\
$\mathrm{C} 4-\mathrm{H} 4$ & 0.9300 & $\mathrm{C} 12-\mathrm{H} 12 \mathrm{~B}$ & 0.9600 \\
$\mathrm{C} 5-\mathrm{C} 6$ & $1.379(9)$ & $\mathrm{C} 12-\mathrm{H} 12 \mathrm{~A}$ & 0.9600 \\
$\mathrm{C} 6-\mathrm{C} 7$ & $1.393(9)$ & $\mathrm{C} 12-\mathrm{H} 12 \mathrm{C}$ & 0.9600 \\
$\mathrm{C} 6-\mathrm{H} 6$ & 0.9300 & $\mathrm{C} 13-\mathrm{H} 13 \mathrm{~A}$ & 0.9600 \\
$\mathrm{C} 7-\mathrm{C} 11$ & $1.542(9)$ & $\mathrm{C} 13-\mathrm{H} 13 \mathrm{~B}$ & 0.9600 \\
$\mathrm{C} 8-\mathrm{C} 9$ & $1.501(12)$ & $\mathrm{C} 13-\mathrm{H} 13 \mathrm{C}$ & 0.9600 \\
$\mathrm{C} 8-\mathrm{C} 10$ & $1.507(10)$ & &
\end{tabular}




\begin{tabular}{|c|c|c|c|}
\hline $\mathrm{C} 1-\mathrm{N} 1-\mathrm{C} 2$ & $118.9(5)$ & $\mathrm{C} 8-\mathrm{C} 9-\mathrm{H} 9 \mathrm{~B}$ & 109.5 \\
\hline $\mathrm{N} 1-\mathrm{C} 1-\mathrm{C} 1^{\mathrm{i}}$ & $120.3(7)$ & $\mathrm{H} 9 \mathrm{C}-\mathrm{C} 9-\mathrm{H} 9 \mathrm{~B}$ & 109.5 \\
\hline $\mathrm{N} 1-\mathrm{C} 1-\mathrm{H} 1$ & 119.9 & $\mathrm{C} 8-\mathrm{C} 9-\mathrm{H} 9 \mathrm{~A}$ & 109.5 \\
\hline $\mathrm{C} 1{ }^{\mathrm{i}}-\mathrm{C} 1-\mathrm{H} 1$ & 119.9 & $\mathrm{H} 9 \mathrm{C}-\mathrm{C} 9-\mathrm{H} 9 \mathrm{~A}$ & 109.5 \\
\hline $\mathrm{C} 7-\mathrm{C} 2-\mathrm{C} 3$ & $122.3(5)$ & $\mathrm{H} 9 \mathrm{~B}-\mathrm{C} 9-\mathrm{H} 9 \mathrm{~A}$ & 109.5 \\
\hline $\mathrm{C} 7-\mathrm{C} 2-\mathrm{N} 1$ & $119.3(5)$ & $\mathrm{C} 8-\mathrm{C} 10-\mathrm{H} 10 \mathrm{C}$ & 109.5 \\
\hline $\mathrm{C} 3-\mathrm{C} 2-\mathrm{N} 1$ & $118.4(5)$ & $\mathrm{C} 8-\mathrm{C} 10-\mathrm{H} 10 \mathrm{~A}$ & 109.5 \\
\hline $\mathrm{C} 4-\mathrm{C} 3-\mathrm{C} 2$ & $118.2(5)$ & $\mathrm{H} 10 \mathrm{C}-\mathrm{C} 10-\mathrm{H} 10 \mathrm{~A}$ & 109.5 \\
\hline $\mathrm{C} 4-\mathrm{C} 3-\mathrm{C} 8$ & $120.0(5)$ & $\mathrm{C} 8-\mathrm{C} 10-\mathrm{H} 10 \mathrm{~B}$ & 109.5 \\
\hline $\mathrm{C} 2-\mathrm{C} 3-\mathrm{C} 8$ & $121.8(5)$ & $\mathrm{H} 10 \mathrm{C}-\mathrm{C} 10-\mathrm{H} 10 \mathrm{~B}$ & 109.5 \\
\hline $\mathrm{C} 5-\mathrm{C} 4-\mathrm{C} 3$ & $119.9(6)$ & $\mathrm{H} 10 \mathrm{~A}-\mathrm{C} 10-\mathrm{H} 10 \mathrm{~B}$ & 109.5 \\
\hline $\mathrm{C} 5-\mathrm{C} 4-\mathrm{H} 4$ & 120.1 & $\mathrm{C} 12-\mathrm{C} 11-\mathrm{C} 13$ & $111.5(8)$ \\
\hline $\mathrm{C} 3-\mathrm{C} 4-\mathrm{H} 4$ & 120.1 & $\mathrm{C} 12-\mathrm{C} 11-\mathrm{C} 7$ & $110.3(6)$ \\
\hline $\mathrm{C} 4-\mathrm{C} 5-\mathrm{C} 6$ & $121.9(6)$ & $\mathrm{C} 13-\mathrm{C} 11-\mathrm{C} 7$ & $114.0(6)$ \\
\hline $\mathrm{C} 4-\mathrm{C} 5-\mathrm{Br} 1$ & $119.2(5)$ & $\mathrm{C} 12-\mathrm{C} 11-\mathrm{H} 11$ & 106.9 \\
\hline $\mathrm{C} 6-\mathrm{C} 5-\mathrm{Br} 1$ & $118.9(5)$ & $\mathrm{C} 13-\mathrm{C} 11-\mathrm{H} 11$ & 106.9 \\
\hline $\mathrm{C} 5-\mathrm{C} 6-\mathrm{C} 7$ & $119.9(6)$ & $\mathrm{C} 7-\mathrm{C} 11-\mathrm{H} 11$ & 106.9 \\
\hline $\mathrm{C} 5-\mathrm{C} 6-\mathrm{H} 6$ & 120.1 & $\mathrm{C} 11-\mathrm{C} 12-\mathrm{H} 12 \mathrm{~B}$ & 109.5 \\
\hline $\mathrm{C} 7-\mathrm{C} 6-\mathrm{H} 6$ & 120.1 & $\mathrm{C} 11-\mathrm{C} 12-\mathrm{H} 12 \mathrm{~A}$ & 109.5 \\
\hline $\mathrm{C} 6-\mathrm{C} 7-\mathrm{C} 2$ & $117.7(6)$ & $\mathrm{H} 12 \mathrm{~B}-\mathrm{C} 12-\mathrm{H} 12 \mathrm{~A}$ & 109.5 \\
\hline $\mathrm{C} 6-\mathrm{C} 7-\mathrm{C} 11$ & $120.2(6)$ & $\mathrm{C} 11-\mathrm{C} 12-\mathrm{H} 12 \mathrm{C}$ & 109.5 \\
\hline $\mathrm{C} 2-\mathrm{C} 7-\mathrm{C} 11$ & $122.1(6)$ & $\mathrm{H} 12 \mathrm{~B}-\mathrm{C} 12-\mathrm{H} 12 \mathrm{C}$ & 109.5 \\
\hline $\mathrm{C} 9-\mathrm{C} 8-\mathrm{C} 10$ & $112.5(8)$ & $\mathrm{H} 12 \mathrm{~A}-\mathrm{C} 12-\mathrm{H} 12 \mathrm{C}$ & 109.5 \\
\hline $\mathrm{C} 9-\mathrm{C} 8-\mathrm{C} 3$ & $111.2(6)$ & $\mathrm{C} 11-\mathrm{C} 13-\mathrm{H} 13 \mathrm{~A}$ & 109.5 \\
\hline $\mathrm{C} 10-\mathrm{C} 8-\mathrm{C} 3$ & $113.0(5)$ & $\mathrm{C} 11-\mathrm{C} 13-\mathrm{H} 13 \mathrm{~B}$ & 109.5 \\
\hline $\mathrm{C} 9-\mathrm{C} 8-\mathrm{H} 8$ & 106.5 & $\mathrm{H} 13 \mathrm{~A}-\mathrm{C} 13-\mathrm{H} 13 \mathrm{~B}$ & 109.5 \\
\hline $\mathrm{C} 10-\mathrm{C} 8-\mathrm{H} 8$ & 106.5 & $\mathrm{C} 11-\mathrm{C} 13-\mathrm{H} 13 \mathrm{C}$ & 109.5 \\
\hline $\mathrm{C} 3-\mathrm{C} 8-\mathrm{H} 8$ & 106.5 & $\mathrm{H} 13 \mathrm{~A}-\mathrm{C} 13-\mathrm{H} 13 \mathrm{C}$ & 109.5 \\
\hline $\mathrm{C} 8-\mathrm{C} 9-\mathrm{H} 9 \mathrm{C}$ & 109.5 & $\mathrm{H} 13 \mathrm{~B}-\mathrm{C} 13-\mathrm{H} 13 \mathrm{C}$ & 109.5 \\
\hline $\mathrm{C} 2-\mathrm{N} 1-\mathrm{C} 1-\mathrm{C}^{\mathrm{i}}$ & $-179.3(8)$ & $\mathrm{C} 5-\mathrm{C} 6-\mathrm{C} 7-\mathrm{C} 11$ & $-178.1(6)$ \\
\hline $\mathrm{C} 1-\mathrm{N} 1-\mathrm{C} 2-\mathrm{C} 7$ & $-90.5(7)$ & $\mathrm{C} 3-\mathrm{C} 2-\mathrm{C} 7-\mathrm{C} 6$ & $-3.5(9)$ \\
\hline $\mathrm{C} 1-\mathrm{N} 1-\mathrm{C} 2-\mathrm{C} 3$ & $92.9(7)$ & $\mathrm{N} 1-\mathrm{C} 2-\mathrm{C} 7-\mathrm{C} 6$ & $-179.9(6)$ \\
\hline $\mathrm{C} 7-\mathrm{C} 2-\mathrm{C} 3-\mathrm{C} 4$ & $3.3(8)$ & $\mathrm{C} 3-\mathrm{C} 2-\mathrm{C} 7-\mathrm{C} 11$ & $174.9(5)$ \\
\hline $\mathrm{N} 1-\mathrm{C} 2-\mathrm{C} 3-\mathrm{C} 4$ & $179.8(5)$ & $\mathrm{N} 1-\mathrm{C} 2-\mathrm{C} 7-\mathrm{C} 11$ & $-1.6(8)$ \\
\hline $\mathrm{C} 7-\mathrm{C} 2-\mathrm{C} 3-\mathrm{C} 8$ & $-177.7(5)$ & $\mathrm{C} 4-\mathrm{C} 3-\mathrm{C} 8-\mathrm{C} 9$ & $82.5(8)$ \\
\hline $\mathrm{N} 1-\mathrm{C} 2-\mathrm{C} 3-\mathrm{C} 8$ & $-1.2(8)$ & $\mathrm{C} 2-\mathrm{C} 3-\mathrm{C} 8-\mathrm{C} 9$ & $-96.4(8)$ \\
\hline $\mathrm{C} 2-\mathrm{C} 3-\mathrm{C} 4-\mathrm{C} 5$ & $0.1(9)$ & $\mathrm{C} 4-\mathrm{C} 3-\mathrm{C} 8-\mathrm{C} 10$ & $-45.1(9)$ \\
\hline $\mathrm{C} 8-\mathrm{C} 3-\mathrm{C} 4-\mathrm{C} 5$ & $-178.9(6)$ & $\mathrm{C} 2-\mathrm{C} 3-\mathrm{C} 8-\mathrm{C} 10$ & $135.9(7)$ \\
\hline $\mathrm{C} 3-\mathrm{C} 4-\mathrm{C} 5-\mathrm{C} 6$ & $-3.3(10)$ & $\mathrm{C} 6-\mathrm{C} 7-\mathrm{C} 11-\mathrm{C} 12$ & $108.2(9)$ \\
\hline $\mathrm{C} 3-\mathrm{C} 4-\mathrm{C} 5-\mathrm{Br} 1$ & $177.6(5)$ & $\mathrm{C} 2-\mathrm{C} 7-\mathrm{C} 11-\mathrm{C} 12$ & $-70.1(9)$ \\
\hline $\mathrm{C} 4-\mathrm{C} 5-\mathrm{C} 6-\mathrm{C} 7$ & $3.1(10)$ & $\mathrm{C} 6-\mathrm{C} 7-\mathrm{C} 11-\mathrm{C} 13$ & $-18.1(10)$ \\
\hline $\mathrm{Br} 1-\mathrm{C} 5-\mathrm{C} 6-\mathrm{C} 7$ & $-177.8(5)$ & $\mathrm{C} 2-\mathrm{C} 7-\mathrm{C} 11-\mathrm{C} 13$ & $163.6(7)$ \\
\hline $\mathrm{C} 5-\mathrm{C} 6-\mathrm{C} 7-\mathrm{C} 2$ & $0.3(9)$ & & \\
\hline
\end{tabular}

Symmetry code: (i) $-x+1,-y+1,-z+1$. 


\section{supporting information}

Hydrogen-bond geometry $\left(A,{ }^{\circ}\right)$

\begin{tabular}{lllll}
\hline$D-\mathrm{H} \cdots A$ & $D-\mathrm{H}$ & $\mathrm{H} \cdots A$ & $D \cdots A$ & $D-\mathrm{H} \cdots A$ \\
\hline $\mathrm{C} 8-\mathrm{H} 8 \cdots \mathrm{N} 1$ & 0.98 & 2.40 & $2.880(9)$ & 109 \\
\hline
\end{tabular}

